\title{
Energy Landscape of Ubiquitin Is Weakly Multidimensional
}

\author{
Published as part of The Journal of Physical Chemistry virtual special issue "D. D. Sarma Festschrift". \\ Balaka Mondal, D. Thirumalai, and Govardhan Reddy*
}

Cite This: J. Phys. Chem. B 2021, 125, 8682-8689

Read Online

\section{ACCESS | \\ Џlll Metrics \& More \\ Article Recommendations \\ Supporting Information}

ABSTRACT: Single molecule pulling experiments report timedependent changes in the extension $(X)$ of a biomolecule as a function of the applied force $(f)$. By fitting the data to onedimensional analytical models of the energy landscape, we can extract the hopping rates between the folded and unfolded states in two-state folders as well as the height and the location of the transition state (TS). Although this approach is remarkably insightful, there are cases for which the energy landscape is multidimensional (catch bonds being the most prominent). To assess if the unfolding energy landscape in small single domain proteins could be one-dimensional, we simulated force-induced unfolding of ubiquitin ( $\mathrm{Ub}$ ) using the coarse-grained self-organized

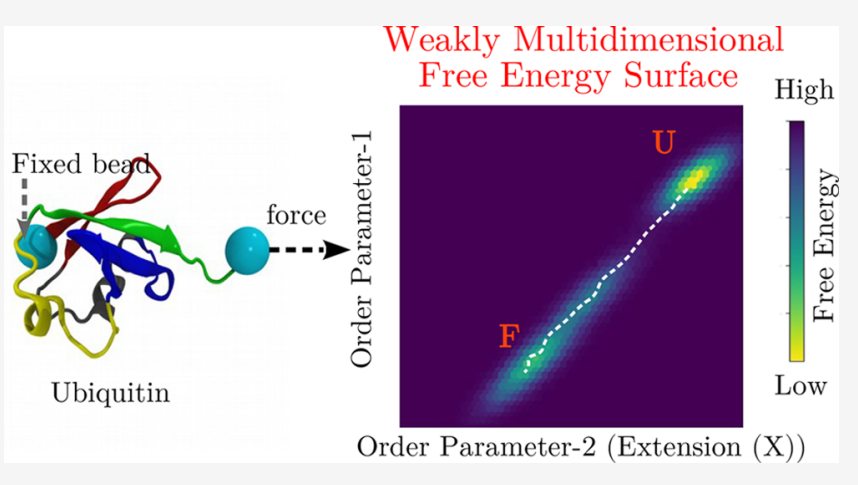
polymer-side chain (SOP-SC) model. Brownian dynamics simulations using the SOP-SC model reveal that the Ub energy landscape is weakly multidimensional (WMD), governed predominantly by a single barrier. The unfolding pathway is confined to a narrow reaction pathway that could be described as diffusion in a quasi-1D $X$-dependent free energy profile. However, a granular analysis using the $P_{\text {fold }}$ analysis, which does not assume any form for the reaction coordinate, shows that $X$ alone does not account for the height and, more importantly, the location of the TS. The $f$-dependent TS location moves toward the folded state as $f$ increases, in accord with the Hammond postulate. Our study shows that, in addition to analyzing the $f$-dependent hopping rates, the transition state ensemble must also be determined without resorting to $X$ as a reaction coordinate to describe the unfolding energy landscapes of single domain proteins, especially if they are only WMD.

\section{INTRODUCTION}

Many cellular functions are triggered by proteins that respond to mechanical stress. ${ }^{1,2}$ In cellular activities, such as muscle contraction and relaxation, cell adhesion, and transport of proteins through a membrane, proteins partially unfold when subject to an external force. ${ }^{3-5}$ These examples illustrate the need to quantitatively understand the molecular basis of the response of biomolecules to forces. Single molecule pulling experiments (optical tweezers, magnetic tweezers, or an atomic force microscope (AFM)), in the constant force mode, measure the extension of the molecule as a function of time, which could be used to sort out the structural transitions in a protein. In the force-ramp experiments, ${ }^{6-11}$ force is increased as a function of time by stretching the protein at a constant loading rate. Typically, but not always, force is applied to the ends of the protein of interest. Pulling at a constant force or constant loading rate unfolds the protein, leading to conformational transitions and eventually complete unfolding at sufficiently large forces. In the constant force mode (force clamp), structural transitions are detected by sudden changes in molecular extension $(X)$ monitored in real time. ${ }^{12}$ By performing the experiments at different values of force $f$, we can extract the force-dependent unfolding rate constant $k_{\mathrm{u}}(f)$. In the force-ramp experiments, ${ }^{13}$ the protein ends are pulled at a constant loading rate, and as $f$ increases, the extension $X$ can be measured. Abrupt changes in the force-extension plots (rips) signify the structural transitions in the protein. In each realization of the experiments, the unfolding force varies stochastically. By performing a large number of experiments, we measure the distribution of rupture forces $p_{\mathrm{u}}(f)$. In principle, the data from the force clamp and constant loading rate modes produce the same information.

The measurements using single molecule pulling experiments have provided a quantitative estimate of the free energy profile (termed free energy surface (FES)) by constructing the histogram of $X$. Phenomenological and analytical models based on Kramer's theory ${ }^{14}$ are routinely used to analyze the data

Received: March 28, 2021

Revised: June 24, 2021

Published: July 28, 2021 
assuming that the folding FES is one-dimensional with $X$ as a reasonable reaction coordinate. The Bell model ${ }^{15}$ assumes that the force-dependent unfolding rate $k_{\mathrm{u}}(f)$ decreases exponentially with the applied force $f$. The fit of the data to the Bell model may be used to extract the intrinsic unfolding rate $k_{\mathrm{u}}(0)$ at $f=0$ and the transition state (TS) location, $\Delta X^{\ddagger}$. A limitation of the Bell model is that it does not relate $k_{\mathrm{u}}(f)$ to the barrier height $\Delta G^{\ddagger}$ or to the shape of the 1D FES. It has become clear that the Bell model is valid only for brittle proteins in which TS does not move with $f .{ }^{16-21}$ However, to account for ductile systems $\left(\Delta X^{\ddagger}\right.$ changes with $\left.f^{22}\right)$, 1D FES (cubic FES or one with a cusp) for which $k_{\mathrm{u}}(f)$ could be analytically calculated have proved to be particularly useful. ${ }^{7,23}$

In an influential article, Dudko, Hummer, and Szabo modeled the FES as linear-cubic and harmonic cusp functions of $X$ and derived analytical expressions ${ }^{24,25}$ for $k_{\mathrm{u}}(f)$ by using a modified Kramer's theory. ${ }^{14}$ The unfolding rate $k_{\mathrm{u}}(f)$ connects $k_{\mathrm{u}}(0)$ to the free energy properties, $\Delta G^{\ddagger}$ and $\Delta X^{\ddagger}$. These quantities are extracted by fitting the data to the theoretical expression. The theory also assumes that FES is onedimensional function of $X$, which might not always hold. Indeed, experiments and simulations provide evidence that the FES for some biomolecules could be multidimensional, ${ }^{26,27}$ involving multiple barriers. ${ }^{28-30}$

If the energy landscape were multidimensional, then it could result in the nonlinearity in $\log k_{\mathrm{u}}(f)$ vs $f$ plot in force-clamp experiments ${ }^{19,31,32}$ or in the most probable rupture force $f^{*}$ vs the logarithm of loading rate $\log r_{\mathrm{f} .}{ }^{28,33-37}$ Is the multidimensionality of the unfolding energy landscape generic? There are only a few single domain proteins for which the presence of multiple unfolding pathways, which requires the use of multidimensionality to explain the experimental data, has been clearly documented. ${ }^{26,30,38-41}$ This suggests that at least strong multidimensionality in single domain proteins may not be the norm but cannot be ruled out unless scrutinized carefully. Ubiquitin ( $\mathrm{Ub}$ ) is an excellent model system to probe the validity of the assumption that the energy landscape is onedimensional due to the availability of extensive single molecule pulling experimental data. ${ }^{42,43}$ To this end, we performed force-clamp and force-ramp pulling simulations of $\mathrm{Ub}$ using the coarse-grained self-organized polymer-side chain (SOPSC) ${ }^{44,45}$ model, which allows us to use forces that are similar to those used in the experiments. We observed a downward curvature in the $\log k_{\mathrm{u}}(f)$ vs $f$ plots in force-clamp simulations, indicating that the FES is weakly multidimensional. ${ }^{26}$ The nonlinearity observed in both the force-clamp and force-ramp simulation data due to the movement of the TS toward the folded state accords well with the Hammond postulate. The location of the TS extracted from the experiments does not satisfy the $P_{\text {fold }}$ criterion. ${ }^{46}$ Taken together, our work shows that, in addition to fitting experimentally measured rates to theories that assume that the free energy profile is onedimensional, additional tests that do not rely on an assumed reaction coordinate or experimental data may be needed to understand the force-induced unfolding dynamics.

\section{METHODS}

SOP-SC Model and Simulation Method. Brownian dynamics simulations at temperatures $T=300$ and $332 \mathrm{~K}$ are performed ${ }^{47}$ using the SOP-SC model. ${ }^{44,45}$ The interactions between the side chains are given by the statistical potential. ${ }^{48}$ The SOP-SC model and the simulation method$\operatorname{ology}^{49,50}$ are described in detail in the Supporting
Information. The SOP-SC energy function parameters are given in Tables $\mathrm{S} 1-\mathrm{S} 3$.

In the force-clamp simulations, we fixed the $N$-terminal end of $\mathrm{Ub}$ (backbone bead of residue 1) and applied a constant force $f$ to the $C$-terminal end (backbone bead of residue 76). In the force-ramp simulations, the backbone bead of residue 1 is fixed, and the backbone bead of residue 76 is attached to a spring, with a spring constant $k_{\mathrm{s}}=35 \mathrm{pN} / \mathrm{nm}$. The other end of the spring is moved at a constant velocity $v$ to ramp up the force to unfold the protein. The loading rate, $r_{\mathrm{f}}=k_{\mathrm{s}} v$, ranges from $8.75 \times 10^{4}$ to $8.75 \times 10^{7} \mathrm{pN} / \mathrm{s}$. The end-to-end distance, $X$, of $\mathrm{Ub}$ is the distance between the backbone beads of residues 1 and 76 . The structural overlap function, ${ }^{51} \chi$, is defined as $\chi=1-\left(1 / N_{\text {tot }}\right) \sum_{i=1}^{N_{\text {tot }}} \Theta\left(\delta-\left|r_{i}-r_{i}^{0}\right|\right)$ to distinguish between different populated states of the protein. Here, $N_{\text {tot }}(=$ $11026)$ is the number of pairs of interaction centers in the SOP-SC model of $\mathrm{Ub}$ assuming that at least two bonds separate the interaction centers, $r_{i}$ is the distance between the $i$ th pair of beads, and $r_{i}^{0}$ being the corresponding distance in the folded state, $\Theta$ is the Heaviside step function, and $\delta=2 \AA$.

Transition State Ensemble. The numerically precise transition state ensemble (TSE) is identified from the unfolding trajectories obtained at different $f$ values at $T=$ 300 and $332 \mathrm{~K}$ by using the $P_{\text {fold }}$ analysis. ${ }^{46}$ Putative TSE structures are picked from the region in the unfolding trajectories where the folded state starts to unravel. This region satisfies the conditions $0.66<\chi<0.68$ and $-50.0 \mathrm{kcal} /$ $\mathrm{mol}<E_{\mathrm{CG}}<-40.0 \mathrm{kcal} / \mathrm{mol}$, where $E_{\mathrm{CG}}$ is the potential energy of a protein conformation in the SOP-SC representation. To compute the commitment probability $P_{\text {fold }}$ for the probable TSEs, 500 short Brownian dynamics simulations are spawned with the friction value $\zeta=1.0 \mathrm{~m} / \tau_{\mathrm{H}}$, where $m$ is the mass of the bead and $\tau_{\mathrm{H}}$ is the time unit of the Brownian time step. If the $P_{\text {fold }}$ of a structure is in the range $0.4<P_{\text {fold }}<0.6$, then the structure is classified as belonging to the TSE. ${ }^{46}$

\section{RESULTS AND DISCUSSION}

Force-Clamp Pulling Simulations of Ub. We first performed force-clamp simulations by applying a constant $f$ to the protein ends. At $T=332 \mathrm{~K}$ and $f=0 \mathrm{pN}$, the free energy difference $(\Delta G)$ between the Ub folded and unfolded states is $\approx 6.6 \mathrm{kcal} / \mathrm{mol}$. The good agreement between the calculated and the experimental values ${ }^{32}$ for $\Delta G$ provides a measure of validation of the SOP-SC model. Upon application of $f$, the end-to-end distance of the protein, $X$ increased by $\approx 22 \mathrm{~nm}$, in agreement with experiments ${ }^{43}$ and previous simulations ${ }^{53-60}$ (Figures S1 and S2).

Ub Unfolding Is Weakly Multidimensional. Forceinduced unfolding of Ub occurs in a two-state manner without populating any intermediates (Figure S1). We observed a single dominant unfolding pathway of $\mathrm{Ub}$ at $T=300$ and 332 $\mathrm{K}$, at all forces (Figure S2). At each $f$, we performed 100 independent pulling simulations to compute the average unfolding time $\langle\tau(f)\rangle$. Assuming two-state kinetics, the forcedependent unfolding rate is taken to be $k_{\mathrm{u}}(f)=\langle\tau(f)\rangle^{-1}$. The relative location of the TS, $\Delta X^{\ddagger}$, may be obtained by fitting the simulation data in the $\left[f, \log \left(k_{\mathrm{u}}(f)\right)\right]$ plot to the Bell model ${ }^{15}$

$$
k_{\mathrm{u}}(f)=k_{\mathrm{u}}(0) \exp \left(f \Delta X^{\ddagger} / k_{\mathrm{B}} T\right)
$$

where $k_{\mathrm{B}}$ is the Boltzmann constant and $\Delta X^{\ddagger}=X^{\ddagger}-X^{\mathrm{F}}$, where $X^{\ddagger}$ is the end-to-end distance of $\mathrm{Ub}$ in the TS and $X^{\mathrm{F}}$ is the end-to-end distance of $\mathrm{Ub}$ in the folded state at $f=0 \mathrm{pN}$. The 
fit of $\left[f, \log \left(k_{\mathrm{u}}(f)\right)\right]$ to eq 1 at $T=332 \mathrm{~K}$ yields $\Delta X^{\ddagger} \approx 0.24 \pm$ $0.04 \mathrm{~nm}$, which agrees with the AFM pulling experiments at high forces, ${ }^{43,61-63}$ and $k_{\mathrm{u}}(0)=2.1 \pm 0.33 \mathrm{~s}^{-1}$ (Figure 1). The fitting procedure is described in the Supporting Information.
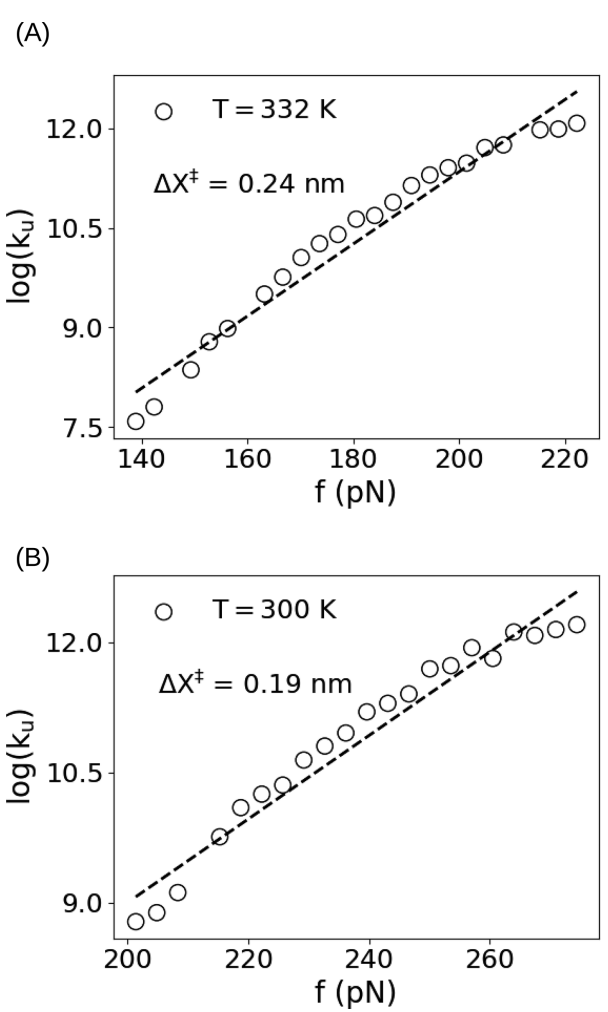

Figure 1. Force-dependent unfolding rate, $k_{w}$ computed by using the force-clamp mode at (A) $T=332 \mathrm{~K}$ and (B) $T=300 \mathrm{~K}$. The dashed line is a fit to the Bell model. At $T=332 \mathrm{~K}(300 \mathrm{~K})$, the extracted $\Delta X^{\ddagger}=0.24 \mathrm{~nm}(0.19 \mathrm{~nm})$ and $k_{\mathrm{u}}(0)=2.1 \pm 0.33 \mathrm{~s}^{-1}(0.53 \pm 0.12$ $\left.\mathrm{s}^{-1}\right)$. Deviation from linearity is observed, especially at higher forces leading to a downward curvature.

Although at lower forces $(\lesssim 170 \mathrm{pN}, T=332 \mathrm{~K})$ the plot is linear, deviation from linearity is observed for higher forces leading to a downward curvature. The Bell model predicts a constant slope $\left(\frac{\Delta X^{\ddagger}}{k_{\mathrm{B}} T}\right)$ for the $\left[f, \log \left(k_{\mathrm{u}}(f)\right)\right]$ plot based on the assumption that TS location is independent of the pulling force. However, curvatures are often observed at high forces either due to force-dependent switch in the unfolding pathways involving different TSs ${ }^{31,64}$ or due to the dependence of the TS on the applied force. ${ }^{22} \mathrm{~A}$ decrease in $\Delta X^{\ddagger}$ at higher values of force (in agreement with Hammond's postulate) can lead to a minor downward curvature (Figure 1). We computed residuals (= observed value - fitted value) to determine whether a linear or nonlinear model better fits the data set. Randomness in the residuals (errors) is a measure of how well a model fits the given data set. The residuals obtained from the Bell model fit to force-clamp data at $T=300$ and $332 \mathrm{~K}$ formed a curved pattern, which indicates that linear models are not suitable to fit the data (Figure S3). Although randomness in the residuals is a criterion for selecting the suitable model, it does not necessarily ensure the adequacy of the model.

We believe that the downward curvature is due to the weak multidimensionality ${ }^{26}$ (WMD) of the FES. We refer to this FES $F(\mathbf{r}, X)$ (where $\mathbf{r}$ represents conformational degrees of freedom different from $X$ ) as weakly multidimensional ${ }^{26}$ because the unfolding process is confined to a single, narrow reaction pathway in the multidimensional space and the absence of parallel unfolding pathways (Figure S2). A previous computational study ${ }^{65}$ using an all-atom model also suggested that the $\mathrm{Ub}$ unfolding landscape could be multidimensional. In WMD landscapes, $k_{\mathrm{u}}(f)$ satisfies the conditions $\frac{\mathrm{d} \log k_{\mathrm{u}}}{\mathrm{d} f} \geq 0$ and $\frac{\mathrm{d}^{2} \log k_{u}}{\mathrm{~d} f^{2}} \leq 0$, which are the conditions for downward curvature, and $\mathrm{Ub}$ satisfies them at high $f$ values (Figure 1). A minor downward curvature in a WMD energy landscape suggests a force-dependent movement of the TSE (see below).

Unfolding Involves Crossing a Single Major Barrier. We also performed force-ramp simulations by pulling one end of $\mathrm{Ub}$ with a constant velocity at $T=332 \mathrm{~K}$. The average force at which Ub unfolds, $\left\langle f_{\mathrm{u}}\right\rangle$, depends linearly ${ }^{53,66}$ on the logarithm of the loading rate, $\log \left(r_{\mathrm{f}}\right)$, for loading rates $r_{\mathrm{f}} \leq$ $8.75 \times 10^{7} \mathrm{pN} / \mathrm{s}$ and deviates from linearity at larger $r_{\mathrm{f}}$ (Figure 2 and Figure S4). Assuming that the Bell model is valid, Evans

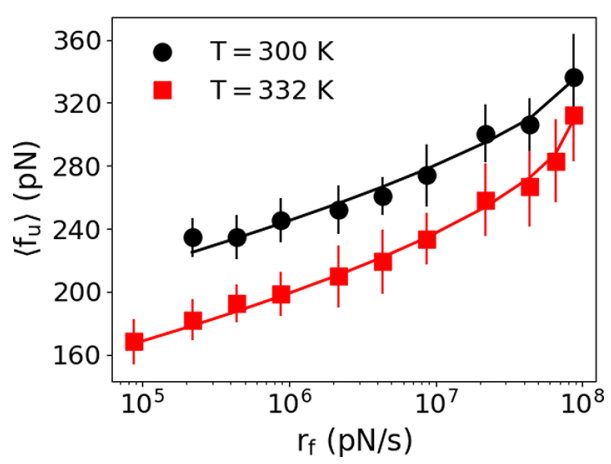

Figure 2. Average unfolding force $\left\langle f_{\mathrm{u}}\right\rangle$ is plotted as a function of logloading rate $\log \left(r_{\mathrm{f}}\right)$. Solid lines are fit of the data to eq 3 . At $T=332 \mathrm{~K}$ $(300 \mathrm{~K})$, the extracted parameters are $\nu=0.58 \pm 0.0947(0.69 \pm$ $0.29), \Delta X^{\ddagger}=0.66 \pm 0.19 \mathrm{~nm}(0.54 \pm 0.46 \mathrm{~nm}), \Delta G^{\ddagger}=26.07 \pm 4.49$ $k_{\mathrm{B}} T\left(30.0 \pm 15.36 k_{\mathrm{B}} T\right)$, and $\kappa=1.27 \times 10^{7} \pm\left(3.95 \times 10^{6}\right) \mathrm{s}^{-1}(1.15$ $\left.\times 10^{7} \pm\left(1.02 \times 10^{7}\right) \mathrm{s}^{-1}\right)$.

and Ritchie ${ }^{67,68}$ derived an equation for the most probable unfolding force $f^{*}$ :

$$
f^{*}=\frac{k_{\mathrm{B}} T}{\Delta X^{\ddagger}} \log \left(\frac{r_{\mathrm{f}} \Delta X^{\ddagger}}{k_{\mathrm{u}}(0) k_{\mathrm{B}} T}\right)
$$

If $\left\langle f_{\mathrm{u}}\right\rangle \approx f^{*}$, we can estimate $\Delta X^{\ddagger}$ from the slope of the linear region in the $\left[\log r_{\mathrm{f}},\left\langle f_{\mathrm{u}}\right\rangle\right]$ plot. The values of $\Delta X^{\ddagger}$ extracted by fitting only the linear regime in the plots at $T=332$ and $300 \mathrm{~K}$ are 0.34 and $0.38 \mathrm{~nm}$, respectively (Figure S4). These values are in reasonable agreement with $\Delta X^{\ddagger}=0.24 \mathrm{~nm}$ (Figure 1). We also determined the TSE using the $P_{\text {fold }}$ method for the lowest loading rate, $r_{\mathrm{f}}=8.75 \times 10^{4} \mathrm{pN} / \mathrm{s}$, accessible in simulations at $T=332 \mathrm{~K}$ (Figure S5). The value of $\Delta X^{\ddagger}$ computed from the TSE is $1.43 \mathrm{~nm}$, and it is in good agreement with the result from constant force simulations (Figure 3) but deviates from the values extracted from the Bell model analysis.

The deviation from linearity, with an upward curvature, in the $\left[\log r_{\mathrm{f}},\left\langle f_{\mathrm{u}}\right\rangle\right]$ plots is evidence for the movement of the TS with force. ${ }^{69}$ To interpret the curvature in $\log r_{\mathrm{f}} \mathrm{vs}\left\langle f_{\mathrm{u}}\right\rangle$ plot, a theoretical model ${ }^{17}$ could be used to assess if unfolding occurs by crossing a single barrier or multiple barriers in the one- 
(A)

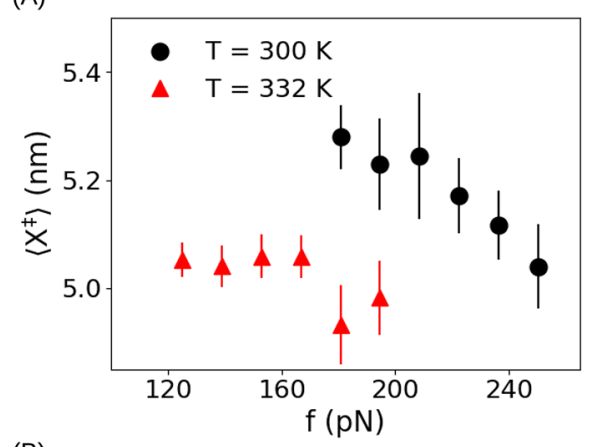

(B)

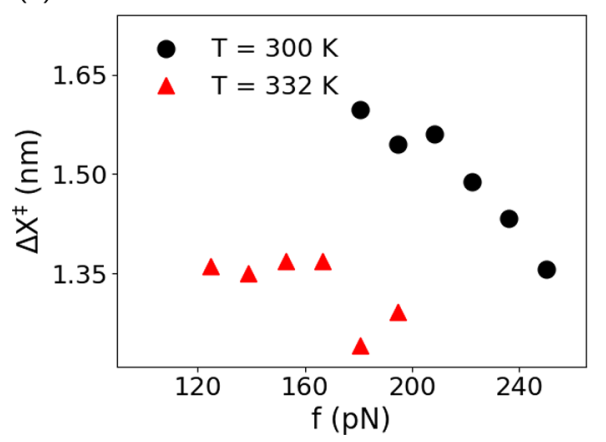

Figure 3. (A) Average end-to-end distance of the Ub conformations in the TSE, $\left\langle X^{\ddagger}\right\rangle$, as a function of the pulling force $f$. Data shown in circles and triangles are obtained at $T=300$ and $332 \mathrm{~K}$, respectively. (B) Relative location of the TS from the native state, $\Delta X^{\ddagger}\left(=\left\langle X^{\ddagger}\right\rangle-\right.$ $\left.\left\langle X^{\mathrm{F}}\right\rangle\right)$, as a function of force at $T=300$ and $332 \mathrm{~K}$ in circles and triangles, respectively. The TS location shifts toward the native state as $f$ increases.

dimensional free energy profile. In this model, unfolding transitions occur by thermal activation for $f \ll f_{\mathrm{c}}$ and $\beta \Delta G^{\ddagger} \gg$ 1 , where $f_{\mathrm{c}}$ is the critical force and $\Delta G^{\ddagger}$ is the free energy of activation. The equation for most probable rupture force is given by

$$
f^{*} \approx f_{\mathrm{c}}\left[1-\left(-\frac{k_{\mathrm{B}} T}{\Delta G^{\ddagger}} \log \frac{r_{\mathrm{f}} \Delta X^{\ddagger}}{\kappa k_{\mathrm{B}} T}\right)^{\nu}\right]
$$

where $\kappa$ is the prefactor in Kramer's theory. The parameter $\kappa$ contains information about the curvature of the FES near the folded basin and the barrier. ${ }^{70}$ It estimates the mean first passage time for reaching the TSE from the folded basin under high-friction conditions. Sharp curvatures imply a smaller distance between the basin and the barrier for a fixed barrier height, leading to high transition rates. In Kramer's theory, random multiple-barrier recrossings are allowed, unlike transition state theory (TST), resulting in a lower estimation of transition rate compared to TST. Furthermore, TST provides an upper limit for Kramer's prefactor, which is $\approx \frac{k_{\mathrm{B}} T}{h}$ , where $h$ is Planck's constant. It is known through a series of investigations dating back over two decades that the TST prefactor is simply wrong. For unfolding transitions involving a single barrier crossing, with $f$-dependent TS, the value of the exponent $\nu$ is bound by $\frac{1}{2} \leq \nu \leq 1$, while for transitions with multiple barriers $\nu<\frac{1}{2}$. Assuming that $\left\langle f_{\mathrm{u}}\right\rangle \approx f^{*}$, we fit the data in $\left[\log r_{\mathrm{f}},\left\langle f_{\mathrm{u}}\right\rangle\right]$ plot using eq 3 and obtained $\nu=0.58$ (Figure 2) at $T=332 \mathrm{~K}$. The relative errors from the fit at each data point are shown in Figure S6. The value $\nu=0.58$ suggests that a single barrier crossing explains the force ramp simulations, which also accords well with the force-clamp data. We conclude that the nonlinearity in the $\left[\log r_{\mathrm{f}}\left\langle f_{\mathrm{u}}\right\rangle\right]$ plot is due to the force-dependent movement of the TS (see also Figure 3).

TS Location Depends on $\boldsymbol{f}$. To check whether the TSE movement follows Hammond's postulate, we identified the TSE by using the $P_{\text {fold }}$ method $^{46}$ (see the Methods section) at $T=300$ and $332 \mathrm{~K}$ using the constant force simulation data. The average end-to-end distance of the structures in the TSE, $\left\langle X^{\ddagger}\right\rangle$, varied between $\approx 5.0$ and $5.4 \mathrm{~nm}$ (Figure 3 ). The decrease in $\left\langle X^{\ddagger}\right\rangle$ at the higher temperature is reminiscent of temperature softening demonstrated long ago by using AFM experiments on ddFLN4. ${ }^{71}$ For a fixed $T$, the TS shifts to lower $\left\langle X^{\ddagger}\right\rangle$ values as $f$ increases (Figure 3 ). The average end-to-end distances at $T=300$ and $332 \mathrm{~K}$ in the absence of force $(f=0$ $\mathrm{pN})$ calculated from equilibrium simulations are nearly the same, $\left\langle X^{\mathrm{F}}\right\rangle=3.68$ and $3.70 \mathrm{~nm}$, respectively. ${ }^{50}$ The decrease in $\left\langle\Delta X^{\ddagger}\right\rangle\left(=\left\langle X^{\ddagger}\right\rangle-\left\langle X^{\mathrm{F}}\right\rangle\right)$ as $f$ increases at $T=300 \mathrm{~K}$ shows that the TSE moves closer to the folded state, in agreement with Hammond's postulate, ${ }^{16,69,72}$ which states that the TSE approaches the least stable species in a reaction (Figure 3). In force-induced unfolding, the stability of the native state decreases with an increase in pulling force, which results in the shift of the transition state toward the folded state. The TSE movement also explains the nonlinear behavior in the $[f$, $\left.\log \left(k_{\mathrm{u}}(f)\right)\right]$ plot (Figure 1). We surmise that the gradual TS movement toward the native state with increasing $f$ suggests that there is a single major free energy barrier separating the folded and the unfolded states.

The location of the TS $\left(\Delta X^{\ddagger} \approx 1.5 \mathrm{~nm}\right)$ at $f=195 \mathrm{pN}$ computed directly from the simulations using $P_{\text {fold }}$ analysis, a method that does not rely on a reaction coordinate, differed significantly from the value $\left(\Delta X^{\ddagger} \approx 0.24 \mathrm{~nm}\right)$ extracted by using the Bell fit to either the experimental ${ }^{42,43,62,63}$ or the simulation data. The discordance between the $\Delta X^{\ddagger}$ values indicates that a complete description of the forced-unfolding dynamics requires going beyond one-dimensional free energy profiles.

$X$ Alone Is Not a Good Reaction Coordinate. The disagreement between the $\Delta X^{\ddagger}$ values extracted by using onedimensional free energy profile, with $X$ as the reaction coordinate, and computed directly by using the $P_{\text {fold }}$ analysis raises the possibility that $X$ alone may be an inadequate reaction coordinate for $\mathrm{Ub}$ unfolding. If $X$ were a good reaction coordinate, then the $P_{\text {fold }}$ for the ensemble of structures that are constrained to have $X \approx\left\langle X^{\ddagger}\right\rangle$ should be $\approx 0.5$. At $T=332$ $\mathrm{K}$ and $f=125 \mathrm{pN},\left\langle X^{\ddagger}\right\rangle$ is $5.1 \pm 0.03 \mathrm{~nm}$ (Figure 3).

We picked $200 \mathrm{Ub}$ conformations whose $X$ values are within $5.1 \pm 0.03 \mathrm{~nm}$ and performed $P_{\text {fold }}$ analysis for these structures. The distribution, $P\left(P_{\text {fold }}\right)$, of the $P_{\text {fold }}$ values, shows that a significant fraction of these structures end up in the protein folded state with $P_{\text {fold }}=1$. The rest of the structures lead to approximately a uniform distribution between $0<P_{\text {fold }}<0.9$ (Figure 4). This occurs because most of the Ub conformations with $X$ in the range $\left\langle X^{\ddagger}\right\rangle \pm \sigma$ are closer to the folded basin than the TS. As a result, when short pulling simulations using these conformations are initiated, most of these structures reach the folded basin. We conclude that for $\mathrm{Ub} X$ alone is not a good reaction coordinate, although both the $f$-dependent unfolding rate and the dependence of the mean unfolding force as a function of $r_{\mathrm{f}}$ do not deviate significantly from the expectation based on an effective 1D energy landscape. 


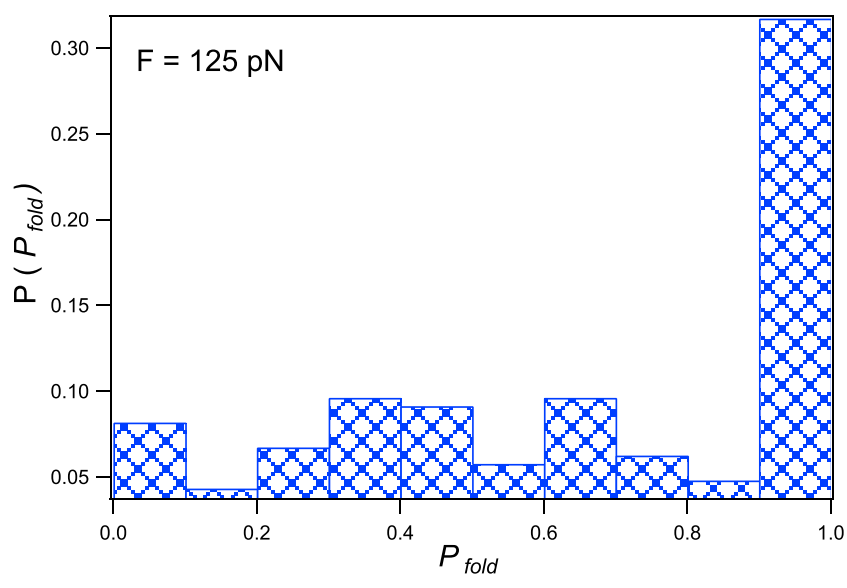

Figure 4. Probability distribution of $P_{\text {fold }}$ values obtained from $200 \mathrm{Ub}$ putative TS structures at $T=332 \mathrm{~K}$ and $f=125 \mathrm{pN}$. The 200 conformations have $X$ within $\left\langle X^{\ddagger}\right\rangle \pm \sigma(5.1 \pm 0.03 \mathrm{~nm})$. Short pulling trajectories spawned by using these structures end up in the folded state basin. The rest of the structures lead to a broad distribution with $P_{\text {fold }}$ between 0 and 0.9. Therefore, from the perspective of the TSE, $X$ alone is not an adequate reaction coordinate.

The probability distribution, $P(X)$, in the absence of $f$, at close to the melting temperature, ${ }^{50} T=353 \mathrm{~K}$, where both the folded and unfolded states are sampled, shows that $X$ does not resolve the folded and unfolded basins of $\mathrm{Ub}$. There is a cusp separating the two basins in the free energy (Figure 5). This finding is also in agreement with the previous simulation result, ${ }^{34}$ which reported that $X$ could be a poor reaction coordinate for $\mathrm{Ub}$ folding.

In single molecule pulling experiments of ductile systems with multidimensional FES, both $\Delta G^{\ddagger}$ and $\Delta X^{\ddagger}$ decrease with the increase in the magnitude of the applied force contrary to the assumptions in the theoretical models (Figure 3 ). In such FES, the molecular extension $X$ alone cannot capture the details of the folding/unfolding process. The fact that we could not identify TS structures by using $P_{\text {fold }}$ analysis when $X$ was chosen to be the only order parameter reinforces the need for a multidimensional treatment of the FES in existing theoretical models. It is possible that a collective coordinate, such as the fraction of native contacts, may be a reasonable choice ${ }^{50,73}$ instead of $X$.

Structural Characterization of the TSE. The transition state structures (TSSs) of Ub in thermal ${ }^{50}$ and force-induced unfolding are identified by using $P_{\text {fold }}$ analysis. ${ }^{46}$ The TSSs are globular and homogeneous, with most of the secondary structural elements and tertiary contacts intact as found in the folded state (Figure 6A), in agreement with $\Psi$-value analysis. ${ }^{7,75}$ Force-induced TSSs consist of a structured Nterminal region with $\alpha_{1}$ helix and $\beta_{1} \beta_{2}$ sheet, in agreement with $\Phi$-value analysis. ${ }^{76}$ The average $\chi$, which gives an estimate of the contact order in the thermal and force-induced TSSs, is $\approx 0.67$ and 0.6, respectively, in agreement with the estimated values for various proteins. ${ }^{77}$ Although the structures are globular, there are some differences in the thermal and forceinduced TSSs (Figure 6B). In the thermal TSSs compared to the folded structure, the contacts between the $\beta$-sheets $\beta_{3} \beta_{5}$ are ruptured completely. Although most of the significant tertiary contacts are present in the force-induced TSSs, the contacts between the sheets $\beta_{1} \beta_{5}$ and $\beta_{3} \beta_{5}$ are weakened considerably compared to the folded state (Figure 6B). In the initial stages of force-induced unfolding, we observe detachment of $\beta_{5}$
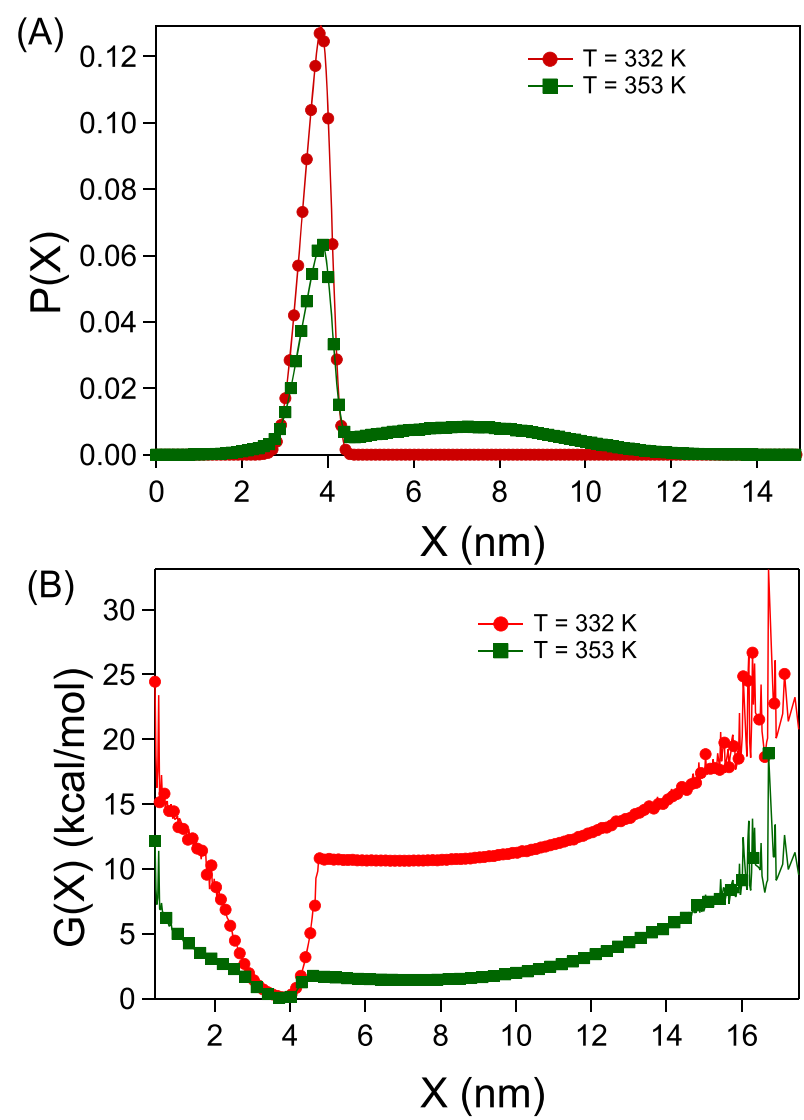

Figure 5. (A) Probability distribution of $X, P(X)$, and (B) FES of Ub projected onto $X, G(X)$, in the absence of external force $(f=0 \mathrm{pN})$ at $T=332$ and $353 \mathrm{~K}$. The melting temperature of $\mathrm{Ub}$ is $\approx 353 \mathrm{~K}$.

strands disrupting contacts with $\beta_{1}$ and $\beta_{3}$ strands (Figure $\mathrm{S} 2 \mathrm{~B}, \mathrm{C})$. This is consistent with the fact that mechanical clamp in $\mathrm{Ub}$ is present between $\beta_{1} \beta_{5}$, the rupture of which constitutes the major energy barrier to unfolding. ${ }^{57,62,78}$ Furthermore, in line with our observation, simulations ${ }^{79}$ also report force induced unfolding of $\mathrm{Ub}$ starting with the rupture of $\beta_{3} \beta_{5}$ contacts.

\section{CONCLUSIONS}

A major advantage of single-molecule pulling experiments is that the energy landscape of proteins can be measured without altering the intraprotein or protein solvent interactions. However, we need useful theoretical models to extract the relevant kinetic properties related to the protein's structural transitions from the experimental data. The common assumptions in the most fruitful models are (1) protein unfolding can be described as a barrier crossing in an effective one-dimensional energy landscape and (2) the location of the transition state is not very sensitive to the applied force. These assumptions need to be tested to ensure that extracted quantities are physically meaningful and consistent with independent experiments and/or reliable simulations.

Unfolding Ub by force, which has been extensively studied by experiments, is a curious case. The dependence of the unfolding rates on force and the mean rupture force as a function of loading rates only exhibit minor deviations from theoretical predictions based on $1 \mathrm{D}$ energy landscape. On the other hand, the structures that are generated by constraining 

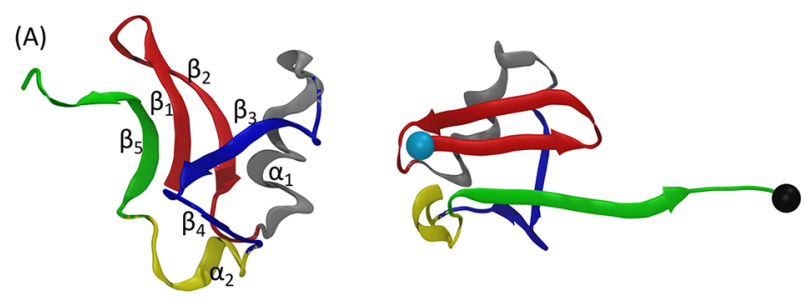

(B)
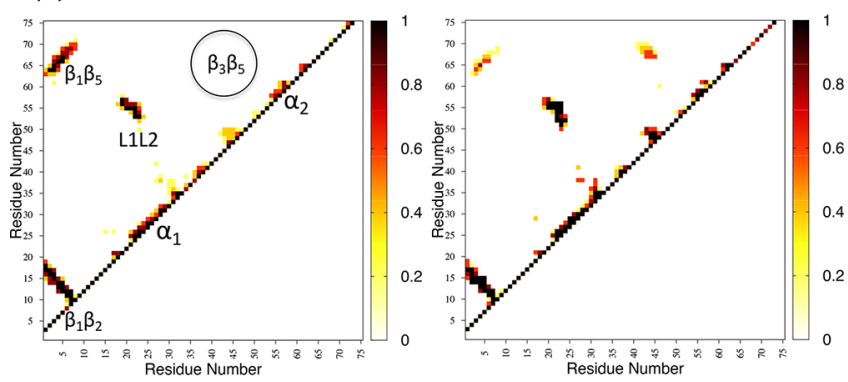

(C)
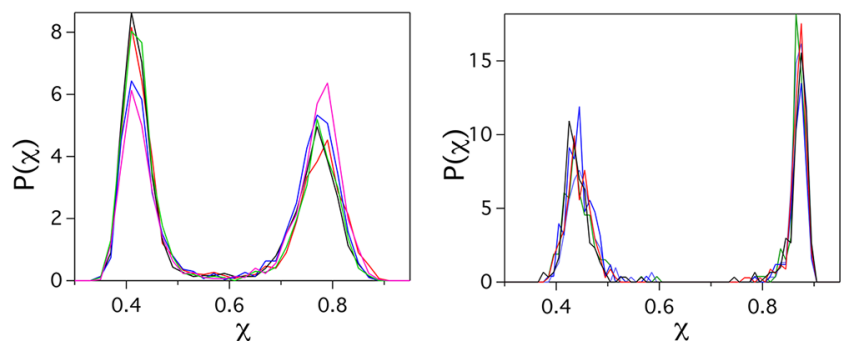

Figure 6. (A) Representative TSSs populated during thermal (left) and force-induced (right) unfolding. The secondary structural elements, five $\beta$ strands $\left(\beta_{1}-\beta_{5}\right)$, and the two $\alpha$ helices $\left(\alpha_{1}\right.$ and $\left.\alpha_{2}\right)$ are shown. (B) Contact map of the thermal TSE (left) shows that contacts between the sheets $\beta_{3} \beta_{5}$ are missing (shown in circle). In the force-induced TSE (right), contacts among the sheets $\beta_{1} \beta_{5}$ and $\beta_{3} \beta_{5}$ are weakened compared to the folded state. (C) Distribution of the final structural overlap parameter, $\chi$, calculated from 500 simulation trajectories spawned by using the TSSs as the initial starting structure. Data are shown for five different structures. The distribution shows that roughly half of these trajectories go into the folded basin and the other half into the unfolded basin, demonstrating that these protein conformations are the TSSs.

the extension to the TS location extracted by fitting the simulation data to the one-dimensional analytical models do not satisfy the $P_{\mathrm{fold}} \approx 0.5$ requirement. By combining both these findings, we demonstrate that the energy landscape of $\mathrm{Ub}$ is weakly multidimensional in which the location of the transition state moves in response to the applied force. Our study shows that only by combining analysis of experimental data using simple theories together with reliable simulations can one learn about the unfolding energy landscape, especially for systems which exhibit weak multidimensionality.

To test the validity of the theoretical models, we compared our simulation results to the AFM pulling experiments of $\mathrm{Ub}$ performed in the high-force regime. ${ }^{42,43,62,63}$ Using optical and magnetic tweezers, it is now possible to perform pulling experiments at low pulling forces $(<1 \mathrm{pN})$ to gain insight into the mechanical properties and force regulation of proteins in the physiologically relevant low-force regime. However, thermodynamic parameters are still extracted by using simplistic theoretical models, which assume a one-dimensional FES with $X$ as the order parameter. Thus, it is essential to improve the existing theoretical models to incorporate features such as the multidimensionality of FES and force-dependent movement of the TS.

\section{ASSOCIATED CONTENT}

\section{SI Supporting Information}

The Supporting Information is available free of charge at https://pubs.acs.org/doi/10.1021/acs.jpcb.1c02762.

Description of the coarse-grained self-organized polymer-side chain protein model and simulation method; Table S1: SOP-SC energy parameter; Table S2: SOP-SC model parameters for ubiquitin; Table S3: side-chain radii of amino acids; Figure S1: multiple independent trajectories showing two-state unfolding of $\mathrm{Ub}$; Figure S2: Ub dominant unfolding pathway; Figure S3: residual plot associated with a Bell fit on force-clamp simulation data of $\mathrm{Ub}$; Figure S4: average unfoding force $\left\langle f_{\mathrm{u}}\right\rangle$ as a function of the loading rate $\ln \left(r_{\mathrm{f}}\right)$; Figure S5: $P_{\text {fold }}$ analysis to identify the TSE from force ramp simulations; Figure S6: relative errors from the fit of eq 3 to the $\left[\log \left(r_{\mathrm{f}}\right),\left\langle f_{\mathrm{u}}\right\rangle\right]$ data (PDF)

\section{AUTHOR INFORMATION}

\section{Corresponding Author}

Govardhan Reddy - Solid State and Structural Chemistry

Unit, Indian Institute of Science, Bengaluru, Karnataka

560012, India; ○ orcid.org/0000-0002-9013-8040;

Phone: +91-80-22933533; Email: greddy@iisc.ac.in;

Fax: $+91-80-23601310$

\section{Authors}

Balaka Mondal - Solid State and Structural Chemistry Unit, Indian Institute of Science, Bengaluru, Karnataka 560012, India

D. Thirumalai - Department of Chemistry, University of

Texas at Austin, Austin, Texas 78712, United States;

(1) orcid.org/0000-0003-1801-5924

Complete contact information is available at:

https://pubs.acs.org/10.1021/acs.jpcb.1c02762

\section{Notes}

The authors declare no competing financial interest.

\section{ACKNOWLEDGMENTS}

A part of this work is funded by the grant to G.R. by the National Supercomputing Mission (MeitY/R\&D/HPC/2(1)/ 2014). D.T. acknowledges grants from the NIH (GM-107703) and the Welch Foundation (Grant F-0019) through the CollieWelch chair. The computations are performed by using the TUE and Cray XC40 clusters at IISc.

\section{REFERENCES}

(1) Bao, G.; Kamm, R. D.; Thomas, W.; Hwang, W.; Fletcher, D. A.; Grodzinsky, A. J.; Zhu, C.; Mofrad, M. R. K. Molecular biomechanics: The molecular basis of how forces regulate cellular function. Cell. Mol. Bioeng. 2010, 3, 91-105.

(2) Sayin, E.; Baran, E. T.; Hasirci, V. Protein-based materials in load-bearing tissue-engineering applications. Regener. Med. 2014, 9, 687-701.

(3) Zhu, C.; Bao, G.; Wang, N. Cell mechanics: Mechanical response, cell adhesion, and molecular deformation. Annu. Rev. Biomed. Eng. 2000, 2, 189-226. 
(4) Bustamante, C.; Chemla, Y.; Forde, N.; Izhaky, D. Mechanical processes in biochemistry. Annu. Rev. Biochem. 2004, 73, 705-748.

(5) Oberhauser, A. F.; Carrion-Vazquez, M. Mechanical biochemistry of proteins one molecule at a time. J. Biol. Chem. 2008, 283, 6617-6621.

(6) Hummer, G.; Szabo, A. Free energy profiles from singlemolecule pulling experiments. Proc. Natl. Acad. Sci. U. S. A. 2010, 107, 21441-21446.

(7) Hummer, G.; Szabo, A. Kinetics from nonequilibrium singlemolecule pulling experiments. Biophys. J. 2003, 85, 5-15.

(8) Kilinc, D.; Lee, G. U. Advances in magnetic tweezers for single molecule and cell biophysics. Integr. Biol. 2014, 6, 27-34.

(9) Yang, B.; Liu, Z.; Liu, H.; Nash, M. A. Next generation methods for single-molecule force spectroscopy on polyproteins and receptorligand complexes. Front. Mol. Biosci. 2020.

(10) Kurland, N. E.; Drira, Z.; Yadavalli, V. K. Measurement of nanomechanical properties of biomolecules using atomic force microscopy. Micron 2012, 43, 116-128.

(11) Deniz, A. A.; Mukhopadhyay, S.; Lemke, E. A. Single-molecule biophysics: at the interface of biology, physics and chemistry. J. $R$. Soc., Interface 2008, 5, 15-45.

(12) Garcia-Manyes, S.; Brujic, J.; Badilla, C. L.; Fernandez, J. M. Force-clamp spectroscopy of single-protein monomers reveals the individual unfolding and folding pathways of I27 and ubiquitin. Biophys. J. 2007, 93, 2436-2446.

(13) Li, P.; Collin, D.; Smith, S.; Bustamante, C.; Tinoco, I. Probing the mechanical folding kinetics of TAR RNA by hopping, force-jump, and force-ramp methods. Biophys. J. 2006, 90, 250-260.

(14) Kramers, H. Brownian motion in a field of force and the diffusion model of chemical reactions. Physica 1940, 7, 284-304.

(15) Bell, G. Models for specific adhesion of cells to cells. Science 1978, 200, 618-627.

(16) Hyeon, C.; Thirumalai, D. Measuring the energy landscape roughness and the transition state location of biomolecules using single molecule mechanical unfolding experiments. J. Phys.: Condens. Matter 2007, 19, 113101-5938.

(17) Hyeon, C.; Thirumalai, D. Multiple barriers in forced rupture of protein complexes. J. Chem. Phys. 2012, 137, 055103.

(18) Cossio, P.; Hummer, G.; Szabo, A. Kinetic ductility and forcespike resistance of proteins from single-molecule force spectroscopy. Biophys. J. 2016, 111, 832-840.

(19) Yuan, G.; Le, S.; Yao, M.; Qian, H.; Zhou, X.; Yan, J.; Chen, H. Elasticity of the transition state leading to an unexpected mechanical stabilization of titin immunoglobulin domains. Angew. Chem., Int. Ed. 2017, 56, 5490-5493.

(20) Marshall, B.; Long, M.; Piper, J.; Yago, T.; McEver, R.; Zhu, C. Direct observation of catch bonds involving cell-adhesion molecules. Nature 2003, 423, 190-193.

(21) Rakshit, S.; Zhang, Y.; Manibog, K.; Shafraz, O.; Sivasankar, S. Ideal, catch, and slip bonds in cadherin adhesion. Proc. Natl. Acad. Sci. U. S. A. 2012, 109, 18815-18820.

(22) Hyeon, C.; Thirumalai, D. Mechanical unfolding of RNA: From hairpins to structures with internal multiloops. Biophys. J. 2007, 92, 731-743.

(23) Dudko, O.; Filippov, A.; Klafter, J.; Urbakh, M. Beyond the conventional description of dynamic force spectroscopy of adhesion bonds. Proc. Natl. Acad. Sci. U. S. A. 2003, 100, 11378-11381.

(24) Dudko, O.; Hummer, G.; Szabo, A. Intrinsic rates and activation free energies from single-molecule pulling experiments. Phys. Rev. Lett. 2006, 96, 1-4.

(25) Dudko, O. K.; Hummer, G.; Szabo, A. Theory, analysis, and interpretation of single-molecule force spectroscopy experiments. Proc. Natl. Acad. Sci. U. S. A. 2008, 105, 15755-15760.

(26) Zhuravlev, P. I.; Hinczewski, M.; Chakrabarti, S.; Marqusee, S.; Thirumalai, D. Force-dependent switch in protein unfolding pathways and transition-state movements. Proc. Natl. Acad. Sci. U. S. A. 2016, 113, E715-E724.

(27) Satija, R.; Berezhkovskii, A. M.; Makarov, D. E. Broad distributions of transition-path times are fingerprints of multi- dimensionality of the underlying free energy landscapes. Proc. Natl. Acad. Sci. U. S. A. 2020, 117, 27116-27123.

(28) Merkel, R.; Nassoy, P.; Leung, A.; Ritchie, K.; Evans, E. Energy landscapes of receptor-ligand bonds explored with dynamic force spectroscopy. Nature 1999, 397, 50-53.

(29) Hyeon, C.; Thirumalai, D. Multiple probes are required to explore and control the rugged energy landscape of RNA hairpins. J. Am. Chem. Soc. 2008, 130, 1538-1539.

(30) Yew, Z. T.; Schlierf, M.; Rief, M.; Paci, E. Direct evidence of the multidimensionality of the free-energy landscapes of proteins revealed by mechanical probes. Phys. Rev. E 2010, 81, 031923.

(31) Jagannathan, B.; Elms, P. J.; Bustamante, C.; Marqusee, S. Direct observation of a force-induced switch in the anisotropic mechanical unfolding pathway of a protein. Proc. Natl. Acad. Sci. U. S. A. 2012, 109, 17820-17825.

(32) Schlierf, M.; Rief, M. Single-molecule unfolding force distributions reveal a funnel-shaped energy landscape. Biophys. J. 2006, 90, L33-L35.

(33) Wojcikiewicz, E. P.; Abdulreda, M. H.; Zhang, X.; Moy, V. T. Force spectroscopy of LFA-1 and its ligands, ICAM-1 and ICAM-2. Biomacromolecules 2006, 7, 3188-3195.

(34) Mathe, J.; Visram, H.; Viasnoff, V.; Rabin, Y.; Meller, A. Nanopore unzipping of individual DNA hairpin molecules. Biophys. J. 2004, 87, 3205-3212.

(35) Porter-Peden, L.; Kamper, S. G.; Wal, M. V.; Blankespoor, R.; Sinniah, K. Estimating kinetic and thermodynamic parameters from single molecule enzyme-inhibitor interactions. Langmuir 2008, 24, 11556-11561.

(36) Friddle, R. W.; Noy, A.; De Yoreo, J. J. Interpreting the widespread nonlinear force spectra of intermolecular bonds (vol 109, pg 13573, 2012). Proc. Natl. Acad. Sci. U. S. A. 2012, 109, 1357313578.

(37) Verbelen, C.; Gruber, H. J.; Dufrene, Y. F. The NTA-HiS(6) bond is strong enough for AFM single-molecular recognition studies. J. Mol. Recognit. 2007, 20, 490-494.

(38) Schlierf, M.; Yew, Z. T.; Rief, M.; Paci, E. Complex unfolding kinetics of single-domain proteins in the presence of force. Biophys. J. 2010, 99, 1620-1627.

(39) Schoenfelder, J.; Perez-Jimenez, R.; Munoz, V. A simple twostate protein unfolds mechanically via multiple heterogeneous pathways at single-molecule resolution. Nat. Commun. 2016, 7, 11777.

(40) Zhuravlev, P. I.; Hinczewski, M.; Thirumalai, D. Low Force unfolding of a single-domain protein by parallel pathways. J. Phys. Chem. B 2021, 125, 1799-1805.

(41) Izadi, D.; Chen, Y.; Whitmore, M. L.; Slivka, J. D.; Ching, K.; Lapidus, L. J.; Comstock, M. J. Combined force ramp and equilibrium high-resolution investigations reveal multipath heterogeneous unfolding of protein G. J. Phys. Chem. B 2018, 122, 11155-11165.

(42) Garcia-Manyes, S.; Dougan, L.; Badilla, C. L.; Brujic, J.; Fernandez, J. M. Direct observation of an ensemble of stable collapsed states in the mechanical folding of ubiquitin. Proc. Natl. Acad. Sci. U. S. A. 2009, 106, 10534-10539.

(43) Schlierf, M.; Li, H.; Fernandez, J. The unfolding kinetics of ubiquitin captured with single-molecule force-clamp techniques. Proc. Natl. Acad. Sci. U. S. A. 2004, 101, 7299-7304.

(44) Hyeon, C.; Dima, R. I.; Thirumalai, D. Pathways and kinetic barriers in mechanical unfolding and refolding of RNA and proteins. Structure 2006, 14, 1633-1645.

(45) Liu, Z.; Reddy, G.; O’Brien, E. P.; Thirumalai, D. Collapse kinetics and chevron plots from simulations of denaturant-dependent folding of globular proteins. Proc. Natl. Acad. Sci. U. S. A. 2011, 108, 7787-7792.

(46) Du, R.; Pande, V.; Grosberg, A.; Tanaka, T.; Shakhnovich, E. On the transition coordinate for protein folding. J. Chem. Phys. 1998, 108, 334-350.

(47) Ermak, D. L.; Mccammon, J. A. Brownian dynamics with hydrodynamic interactions. J. Chem. Phys. 1978, 69, 1352-1360.

(48) Betancourt, M.; Thirumalai, D. Pair potentials for protein folding: Choice of reference states and sensitivity of predicted native 
states to variations in the interaction schemes. Protein Sci. 1999, 8, 361-369.

(49) Reddy, G.; Thirumalai, D. Collapse precedes folding in denaturant-dependent assembly of ubiquitin. J. Phys. Chem. B 2017, 121, 995-1009.

(50) Reddy, G.; Thirumalai, D. Dissecting ubiquitin folding using the self-organized polymer model. J. Phys. Chem. B 2015, 119, $11358-11370$.

(51) Guo, Z.; Thirumalai, D. Kinetics and thermodynamics of folding of a de novo designed four helix bundle. J. Mol. Biol. 1996, 263, 323-343.

(52) Makhatadze, G. Thermodynamics of protein interactions with urea and guanidinium hydrochloride. J. Phys. Chem. B 1999, 103, $4781-4785$.

(53) Pincus, D. L.; Thirumalai, D. Crowding effects on the mechanical stability and unfolding pathways of ubiquitin. J. Phys. Chem. B 2009, 113, 359-368.

(54) Best, R. B.; Paci, E.; Hummer, G.; Dudko, O. K. Pulling direction as a reaction coordinate for the mechanical unfolding of single molecules. J. Phys. Chem. B 2008, 112, 5968-5976.

(55) Das, A.; Mukhopadhyay, C. Mechanical unfolding pathway and origin of mechanical stability of proteins of ubiquitin family: An investigation by steered molecular dynamics simulation. Proteins: Struct., Funct., Genet. 2009, 75, 1024-1034.

(56) Li, M. S.; Kouza, M.; Hu, C.-K. Refolding upon force quench and pathways of mechanical and thermal unfolding of ubiquitin. Biophys. J. 2007, 92, 547-561.

(57) Irback, A.; Mitternacht, S.; Mohanty, S. Dissecting the mechanical unfolding of ubiquitin. Proc. Natl. Acad. Sci. U. S. A. 2005, 102, 13427-13432.

(58) Szymczak, P.; Cieplak, M. Stretching of proteins in a forceclamp. J. Phys.: Condens. Matter 2006, 18, L21-L28.

(59) Kirmizialtin, S.; Huang, L.; Makarov, D. Topography of the free-energy landscape probed via mechanical unfolding of proteins. $J$. Chem. Phys. 2005, 122, 234915.

(60) Li, P.; Makarov, D. Simulation of the mechanical unfolding of ubiquitin: Probing different unfolding reaction coordinates by changing the pulling geometry. J. Chem. Phys. 2004, 121, 4826-4832.

(61) Garcia-Manyes, S.; Brujic, J.; Badilla, C. L.; Fernandez, J. M. Force-clamp spectroscopy of single-protein monomers reveals the individual unfolding and folding pathways of I27 and ubiquitin. Biophys. J. 2007, 93, 2436-2446.

(62) Popa, I.; Fernandez, J. M.; Garcia-Manyes, S. Direct quantification of the attempt frequency determining the mechanical unfolding of ubiquitin protein. J. Biol. Chem. 2011, 286, 3107231079 .

(63) Kuo, T. L.; Garcia-Manyes, S.; Li, J.; Barel, I.; Lu, H.; Berne, B. J.; Urbakh, M.; Klafter, J.; Fernandez, J. M. Probing static disorder in Arrhenius kinetics by single-molecule force spectroscopy. Proc. Natl. Acad. Sci. U. S. A. 2010, 107, 11336-11340.

(64) Guinn, E. J.; Jagannathan, B.; Marqusee, S. Single-molecule chemo-mechanical unfolding reveals multiple transition state barriers in a small single-domain protein. Nat. Commun. 2015, 6, 6861.

(65) Sahoo, A. K.; Bagchi, B.; Maiti, P. K. Unfolding dynamics of ubiquitin from constant force $\mathrm{MD}$ simulation: Entropy-enthalpy interplay shapes the free-energy landscape. J. Phys. Chem. B 2019, 123, 1228-1236.

(66) Evans, E. Energy landscapes of biomolecular adhesion and receptor anchoring at interfaces explored with dynamic force spectroscopy. Faraday Discuss. 1999, 111, 1-16.

(67) Evans, E.; Ritchie, K. Dynamic strength of molecular adhesion bonds. Biophys. J. 1997, 72, 1541-1555.

(68) Evans, E.; Ritchie, K. Strength of a weak bond connecting flexible polymer chains. Biophys. J. 1999, 76, 2439-2447.

(69) Hyeon, C.; Thirumalai, D. Forced-unfolding and force-quench refolding of RNA hairpins. Biophys. J. 2006, 90, 3410-3427.

(70) Hanggi, P.; Talkner, P.; Borkovec, M. Reaction-rate theory - 50 years after kramers. Rev. Mod. Phys. 1990, 62, 251-341.
(71) Schlierf, M.; Rief, M. Temperature softening of a protein in single-molecule experiments. J. Mol. Biol. 2005, 354, 497-503.

(72) Hammond, G. A correlation of reaction rates. J. Am. Chem. Soc. 1955, 77, 334-338.

(73) Cho, S.; Levy, Y.; Wolynes, P. P versus Q: Structural reaction coordinates capture protein folding on smooth landscapes. Proc. Natl. Acad. Sci. U. S. A. 2006, 103, 586-591.

(74) Krantz, B.; Dothager, R.; Sosnick, T. Discerning the structure and energy of multiple transition states in protein folding using psianalysis. J. Mol. Biol. 2004, 337, 463-475.

(75) Krantz, B.; Dothager, R.; Sosnick, T. Discerning the structure and energy of multiple transition states in protein folding using psianalysis (vol 337, pg 463, 2004). J. Mol. Biol. 2005, 347, 1103.

(76) Went, H.; Jackson, S. Ubiquitin folds through a highly polarized transition state. Protein Eng., Des. Sel. 2005, 18, 229-237.

(77) Paci, E.; Lindorff-Larsen, K.; Dobson, C.; Karplus, M.; Vendruscolo, M. Transition state contact orders correlate with protein folding rates. J. Mol. Biol. 2005, 352, 495-500.

(78) Chyan, C.-L.; Lin, F.-C.; Peng, H.; Yuan, J.-M.; Chang, C.-H.; Lin, S.-H.; Yang, G. Reversible mechanical unfolding of single ubiquitin molecules. Biophys. J. 2004, 87, 3995-4006.

(79) Cao, P.; Yoon, G.; Tao, W.; Eom, K.; Park, H. S. The role of binding site on the mechanical unfolding mechanism of ubiquitin. Sci. Rep. 2015, 5, 8757. 